GRADIATION\&APPLICATIONS

ISSN 2466-4294 (online) | rad-journal.org

Vol. 2 | Issue 2 | pp. 145 - 147, 2017

doi: 10.21175/RadJ.2017.02.031

Short note

\title{
RADIOLOGY AND CT SCAN FINDINGS IN PATIENTS WITH SIMULTANEOUS OCCURRENCE OF TUBERCULOSIS AND LUNG CANCER
}

\author{
Marina Marković1,2, Marina Petroviće,,2, \\ Marija Živković Radojević1,2, Aleksandar Dagović1, Vladimir Jurišić² ${ }^{*}$ \\ ${ }^{1}$ Clinical Center Kargujevac, Kragujevac, Serbia \\ ${ }^{2}$ University of Kragujevac, Faculty of Medical Sciences, Kragujevac, Serbia
}

\begin{abstract}
Simultaneous occurrence of lung cancer and pulmonary tuberculosis, as a significant cause of morbidity and mortality, appears in $0.7 \%$ of cases. The mechanisms of interaction between them are not fully clarified. We present a patient who, during the treatment of lung adenocarcinoma, developed pulmonary tuberculosis and, owing to a correct diagnosis, was provided with appropriate treatment. We analyzed the CT and radiographic findings during the follow-up of the patient and discussed the problems and doubts about the diagnosis of simultaneous occurrence of lung cancer and pulmonary tuberculosis. Differential diagnosis between tuberculosis and lung cancer is difficult and can pose a real clinical challenge due to the very similar symptomatology involving fever, malaise, sweating, and loss of body weight. Imaging methods routinely used in clinics, such as RTG, CT, and PET-CT, are of great help in such cases. The existence of the TB infection makes it difficult to adequately determine the nodal status in patients with lung cancer. The newly established lymph nodes may not only be the occurrence of tumors, but also already active tuberculosis or the progression of TB infection. Only the correct diagnosis can lead to successful treatment as described in this case.
\end{abstract}

Key words: Lung cancer, pulmonary tuberculosis, CT scan, diagnosis

\section{INTRODUCTION}

Simultaneous occurrence of lung cancer and pulmonary tuberculosis was first described in 1810 [1] as a significant cause of morbidity and mortality, appearing in $0.7 \%$ of cases [1]. Mechanisms of interaction between them are not fully clarified. Each of these diseases can predispose to the other.

Thus, due to immunosuppression and chronic inflammation, tuberculosis provides fertile ground for the development of lung cancer. Tuberculosis increasingly appears during the treatment of tumors or with the appearance of immune-suppression that exists during the progression of lymphoma, leukemia, lung cancer, head and neck cancer or stomach cancer. Malnutrition, low general conditions and cancer treatment can contribute to the reactivation of old foci and increase the development of tuberculosis [2-6]. Lung cancer increases the susceptibility to the development of tuberculosis and tuberculosis represents an independent negative prognostic factor. Patients suffering from tuberculosis have 11 times more chances of developing lung cancer [6]. Of all histological types which occur with tuberculosis, the most common is lung adenocarcinoma (21.7-56.1\%) [7]. Patients may live for a relatively long period of time if there is a mutation of the EGFR [8]. The greatest risk of tuberculosis exists around 11 months after lung cancer is diagnosed [5].
The initial symptoms of the two diseases are similar. However, if tuberculosis is diagnosed first, radiographic control examinations of patients are performed more often, which increases the likelihood of diagnosing lung cancer [6]. Risk factors for the simultaneous occurrence of these diseases include the male sex, smoking, and older age [7]. The coexistence of tuberculosis and lung cancer in elderly patients is present in $1-2 \%$ of cases [8, 9].

Exceptional similarity of clinical manifestations of the diseases and radiological findings complicates timely diagnosis, especially if both diseases occur simultaneously. They are characterized by the occurrence of cough, fatigue, hemoptysis, weight loss, and anorexia [10]. The clinical and radiographic manifestations are similar, but the treatment and prognosis differ significantly.

The aim of this article is to highlight the problem of setting the differential diagnosis of simultaneous occurrence of lung cancer and tuberculosis with the help of CT scans. We present a patient who, during the treatment of lung adenocarcinoma, developed pulmonary tuberculosis and, owing to a correct diagnosis, was provided with appropriate treatment.

\footnotetext{
*vdvd@lycos.com
} 


\section{CASE REPORT}

The patient, 50 years old, from Kragujevac, was admitted in 2012 with the symptoms including dry cough and fatigue. He had been a smoker for almost 32 years and had smoked up to 60 cigarettes per day. Immediately after hospitalisation, a CT chest scan was done and the results showed a heterogeneous tumor mass in the left lung, measuring $94 \times 65 \mathrm{~mm}$ in size. On both sides, other parts of the pulmonary parenchyma showed diffused oval focal lesions, indicating secondary deposits, but without involving mediastinal lymphadenopathy and pleural spaces. Histology confirmed the diagnosis of adenocarcinoma, a low degree of malignancy and a negative EGFR. The tumor was classified as $\mathrm{T}_{3} \mathrm{Nx} \mathrm{M}$ and the patient was in clinical stage IV, and ECOG performance status was I. After confirming the diagnosis, (Figure 1a, 1b) chemotherapy was applied including the Taxolcarboplatin (CBDCA) protocol for tumor treatment, during 6 cycles.

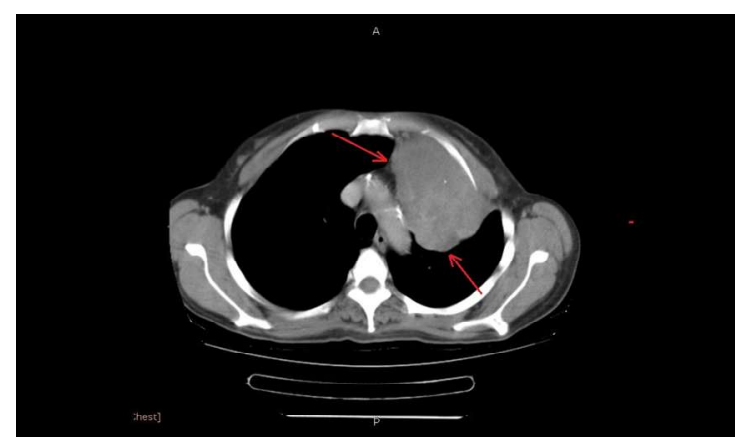

Figure 1a. Primary tumor in 2012

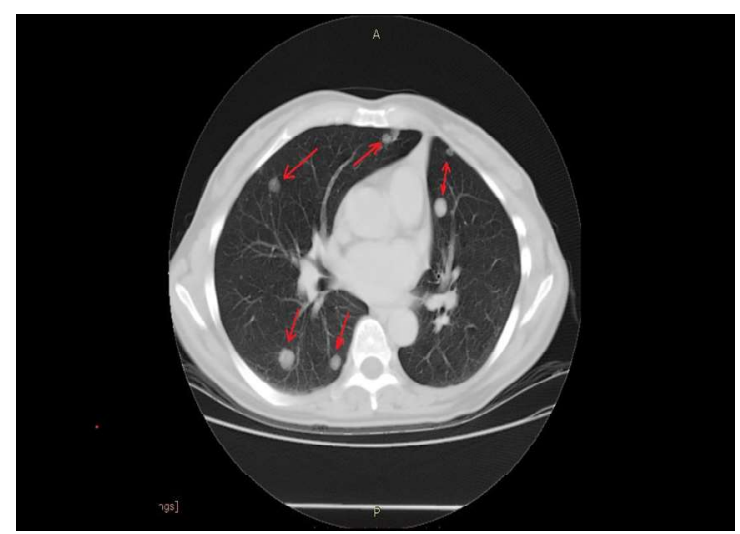

Figure $1 \mathrm{~b}$. Tumor metastasis

After completing the 6 cycles of therapy, a new CT scan showed that the previously described tumor decreased more than $50 \%$ in volume and the focal changes in the lung parenchyma showed also a light regression. After 9 cycles of therapy, a CT lung scan was done again in December 2012 due to elevated body temperature (up to $38^{\circ} \mathrm{C}$ ), productive cough, coughing up yellow mucus, pain in the left hemothorax, and loss of body weight. The CT scan of the lungs at that time showed that previously described changes in the anterior segment of the upper lobe of the left hemothorax were now $50 \times 24 \mathrm{~mm}$ in size, with a peripheral zone pneumonitis in the upper lobe of the left posterolateral hemothorax. In the upper lobe of the lung, the front part of the side close to the pleura contained a consolidated mass with the present hypodense zone that resembled the attenuation of fluid and could have indicated the softening of the tissue (Figure 2a and 2b).

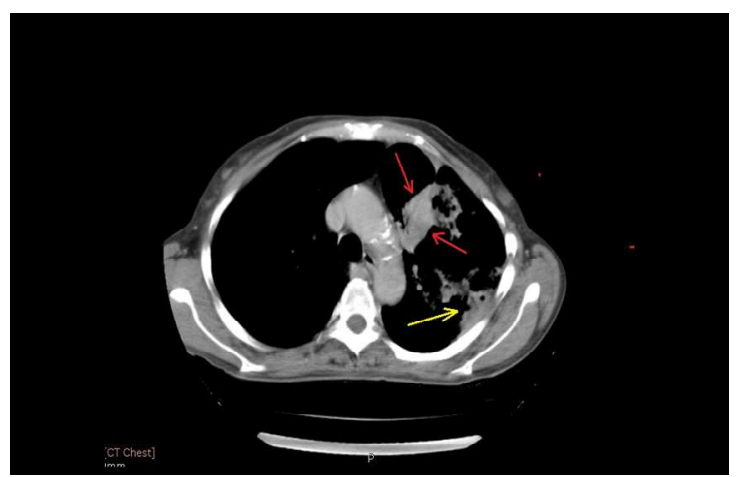

Figure 2a. New lesion with regression of primary tumor and regression of metastasis in 2013

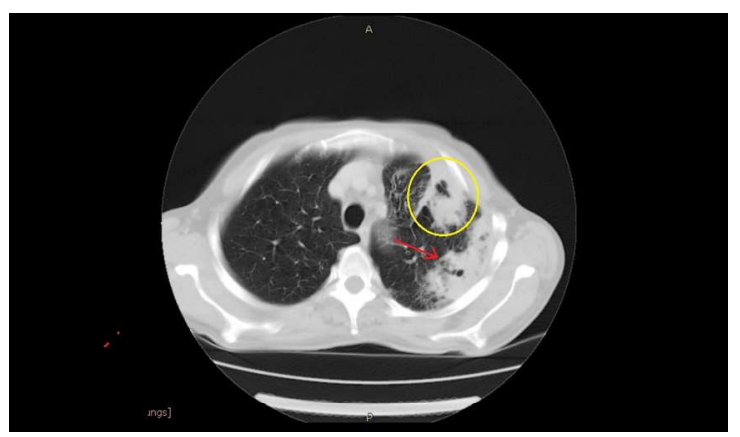

Figure 2b. New lesion in January 2013

Bronchoscopy examination revealed a common finding in the larynx, trachea, and tracheobronchial tree. Cell culture taken from the biopsy of lung tissue was positive for the tuberculosis bacillus and direct scopy revealed the presence of 4 bacilli in 100 investigated fields of view. It was concluded that it was tuberculosis and antituberculotics therapy was immediately started. Six months after the therapy, a CT scan was done again and the results showed that the previously described changes in the lung parenchyma were now stationary characteristics $54 \times 31 \mathrm{~mm}$ in size in the right lung parenchyma and a soft-tissue shadow $23 \times 28 \mathrm{~mm}$ in size. During the routine control (4 years after the first visit), it was concluded that the patient was in good general condition, and ECOG was o.

\section{DISCUSSION}

In this paper, we not only analyzed the CT and radiographic findings during a follow-up of the patient with lung cancer, but also we discussed the problems and doubts about the diagnosis of tuberculosis of the lungs. 
During the treatment of patients with lung cancer, the existence of tuberculosis is rarely suspected, especially in countries with a low incidence of tuberculosis $[1,11]$. Therefore, it is important to educate doctors, especially in smaller centers, to recognize the early signs and symptoms of the two diseases in order for them to be diagnosed in time [12]. The gold standard is a histological confirmation coupled with microbiological analyses. Imaging methods, such as X-ray, CT, and PET-CT, are of invaluable importance for the diagnosis.

The occurrence of the following lesions on CT scans may indicate tuberculosis: parenchymal disease, lymphadenopathy, multilobar infiltration in the upper parts of the lungs, caverns and pleural effusion [2, 10, 11]. Lung cancer is accompanied by the occurrence of peripheral nodular shadows and subpleural consolidation with irregular margins of thin-walled cavities and an elevation of the diaphragm [11, 12, 13]. Regardless of all radiographic differences, lung cancer and tuberculosis cannot be differentiated with certainty [11]. However, the existence of a change in the form of new lesions, segmental or lobar atelectasis, unilateral hilar enlargement, thin-wall cavities, and localized pneumonia may suggest the simultaneous presence of tuberculosis and lung cancer [14]. Adequate treatment, in this case, involves simultaneous complex treatment of malignant and infectious diseases [13].

\section{CONCLUSION}

Differential diagnosis between tuberculosis and lung cancer is difficult and can pose a real clinical challenge due to the very similar symptomatology involving fever, malaise, sweating, and loss of body weight. Imaging methods routinely used in clinics, such as RTG, CT, and PET-CT, are of great help in such cases. The existence of the TB infection makes it difficult to adequately determine the nodal status in patients with lung cancer. The newly established lymph nodes may not only be the occurrence of tumors, but also already active tuberculosis or the progression of TB infection. Only the correct diagnosis can lead to successful treatment as described in this case.

\section{REFERENCES}

1. G. L. Bayle, Recherches sur la phitisie pulmonaire, Paris, France: Galon, 1810. (G. L. Bayle, Research on pulmonary diseases, Paris, France: Galon, 1810).

2. M. Skowroński et al., "Squamous cell lung cancer in a male with pulmonary tuberculosis," Pneumonol. Alergol. Pol., vol. 83, no. 4, pp. 298 - 302, 2015. DOI: 10.5603/PiAP.2015.0049 PMid: 26166791

3. C. H. Chang et al., "Gender-based impact of epidermal growth factor receptor mutation in patients with nonsmall cell lung cancer and previous tuberculosis," Medicine (Baltimore), vol. 94, no. 4, p. e444, Jan. 2015. DOI: 10.1097/MD.0000000000000444

PMid: 25634180

PMCid: PMC4602966
4. V.Y. Su et al., "Latent Tuberculosis Infection and the Risk of Subsequent Cancer," Medicine (Baltimore), vol. 95, no. 4, p. e2352, Jan. 2016.

DOI: 10.1097/MD.0000000000002352

PMid: 26825880

PMCid: PMC5291550

5. G. H. Seo et al., "Cancer-specific incidence rates of tuberculosis: A 5-year nationwide population-based study in a country with an intermediate tuberculosis burden," Medicine (Baltimore), vol. 95, no. 38, p. e4919, Sep. 2016.

DOI: 10.1097/MD.0000000000004919

PMid: 27661041 PMCid: PMC5044911

6. D. R. Silva et al., "Pulmonary tuberculosis and lung cancer: simultaneous and sequential occurrence," J. Bras. Pneumol, vol. 39, no. 4, pp. $484-489$, Jun-Aug. 2013.

DOI: $10.1590 / \mathrm{S} 180637132013000400013$

PMid: 24068271 PMCid: PMC4075860

7. N. A. Yener et al., "Primary Pulmonary Mucinous (Colloid) Adenocarcinoma that Arose in the Cavernomyoplasty Area in a Patient with Tuberculosis: A Rare Case Report," Turk. J. Pathol., vol. 33, no. 1, pp. 062 - 065, Feb. 2017.

DOI: $10.5146 /$ tjpath.2014.01228

8. Y. Zhou et al., "The presence of old pulmonary tuberculosis is an independent prognostic factor for squamous cell lung cancer survival," $J$. Cardiothorac. Surg, vol. 8, no. 123, May 2013. DOI: $10.1186 / 1749-8090-8-123$

9. Y. Wanget al., "Coexistence of acute miliary pulmonary tuberculosis and metastatic lung adenocarcinoma: a case report," Quant. Imaging. Med. Surg., vol. 3, no. 3, pp. $178-179$, Jun. 2013.

DOI: $10.3978 / \mathrm{j}$.issn.2223-4292.2013.06.06

PMid: 23833731

PMCid: PMC 3701099

10. M. Bhatt et al., "Pulmonary tuberculosis as differential diagnosis of lung cancer," South Asian J. Cancer, vol. 1, no. 1, pp. 36 - 42, Jul. 2012.

DOI: $10.4103 / 2278-330 X .96507$

PMid: 24455507

PMCid: PMC 3876596

11. I. Hammen, "Tuberculosis mimicking lung cancer," Respir. Med. Case Rep., vol. 16, no. 45 - 47, Jul. 2015. DOI: 10.1016/j.rmcr.2015.06.007

12. K. Ramachandran et al., "Physician Related Delays in the Diagnosis of Lung Cancer in India," J. Clin. Diagn. Res., vol. 10, no. 11, pp. OCo5 - OCo8, Nov. 2016. DOI: $10.7860 / J C D R / 2016 / 22737.8823$

13. W. Zhao et al., "Differential diagnosis of acute miliary pulmonary tuberculosis from widespread-metastatic cancer for postoperative lung cancer patients: two cases," J. Thorac. Dis, vol. 9, no. 2, pp. E115 - E120, Feb. 2017.

DOI: 10.21037/jtd.2017.02.13

PMid: 28275493 PMCid: PMC5334083

14. M. Hara et al., "Carcinomatous pleuritis and pericarditis accompanied by pulmonary tuberculosis," Respirol. Case Rep, vol. 4, no. 6, p. e00202, Oct. 2016. DOI: $10.1002 /$ rcr2.202

PMid: 28031837 PMCid: PMC5167291 